\title{
Resource allocation varies with parental sex and brood size in the asynchronously hatching green-rumped parrotlet (Forpus passerinus)
}

\author{
Amber E. Budden • Steven R. Beissinger
}

Received: 9 October 2008 / Revised: 8 December 2008 / Accepted: 9 December 2008 / Published online: 14 January 2009

(C) The Author(s) 2008. This article is published with open access at Springerlink.com

\begin{abstract}
When eggs hatch asynchronously, offspring arising from last-hatched eggs often exhibit a competitive disadvantage compared with their older, larger nestmates. Strong sibling competition might result in a pattern of resource allocation favoring larger nestlings, but active food allocation towards smaller offspring may compensate for the negative effects of asynchronous hatching. We examined patterns of resource allocation by greenrumped parrotlet parents to small and large broods under control and food-supplemented conditions. There was no difference between parents and among brood sizes in visit rate or number of feeds delivered, although females spent marginally more time in the nest than males. Both male and female parents preferentially fed offspring that had a higher begging effort than the remainder of the brood. Mean begging levels did not differ between small and large broods, but smaller offspring begged more than their older nestmates in large broods. Male parents fed small offspring less often in both brood sizes. Female parents fed offspring evenly in small broods, while in large broods they fed smaller offspring more frequently, with the exception of the very last hatched individual. These data suggest male parrotlets exhibit a feeding
\end{abstract}

Communicated by M. Leonard

A. E. Budden $\cdot$ S. R. Beissinger

Ecosystem Sciences Division,

Department of Environmental Science,

Policy \& Management, University of California,

137 Mulford Hall,

Berkeley, CA 94720-3114, USA

Present address:

A. E. Budden $(\bowtie)$

National Center for Ecological Analysis and Synthesis,

735 State Street, Suite 300,

Santa Barbara, CA 93101-3351, USA preference for larger offspring-possibly arising from the outcome of sibling competition-but that females practice active food allocation, particularly in larger brood sizes. These differential patterns of resource allocation between the sexes are consistent with other studies of parrots and may reflect some level of female compensation for the limitations imposed on smaller offspring by hatching asynchrony.

Keywords Hatching asynchrony · Honest signaling · Begging behavior $\cdot$ Sibling competition $\cdot$ Food allocation

\section{Introduction}

Many avian species initiate incubation before completion of the clutch, resulting in hatching asynchrony (Clark and Wilson 1981; Stoleson and Beissinger 1995). Consequently, offspring arising from last-hatched eggs may exhibit a competitive disadvantage compared with their older, larger nestmates, and these individuals can have a reduced probability of survival (Magrath 1990). The adaptive value of hatching asynchrony is unclear and may vary according to species' life history traits (Stoleson and Beissinger 1995). Given limited empirical support for fitness advantages from hatching asynchrony (Nilsson 1995; Stoleson and Beissinger 1995; Stoleson and Beissinger 1997), alternative hypotheses have focused on the benefits of the early onset of incubation for egg viability (Veiga 1992; Stoleson and Beissinger 1999; Beissinger et al. 2005; Cook et al. 2005) and for protecting eggs from predators, brood parasites, or nest competitors (e.g. Bollinger et al. 1990; Romagnano et al. 1990; Beissinger et al. 1998). Consequently, parents may attempt to mitigate the negative effects of hatching asynchrony imposed by the necessity to initiate 
incubation early. Such compensatory mechanisms could include selectively advantaging later-hatched offspring through increased egg mass with laying sequence (Clark and Wilson 1981; Budden and Beissinger 2005), differential allocation of maternal hormones (Muller et al. 2007) or selective feeding of later-hatched young (Krebs 2002).

Honest signaling models predict that variation in nestling begging behavior corresponds directly to individual need (Godfray 1991; Godfray 1995) and that parents respond to these begging signals (e.g., nestling posture and stretching, gaping, and vocalizations, reviewed in Budden and Wright 2001). However, body-size variation arising from hatching asynchrony can play an important role in sibling competition, with relatively larger nestlings gaining a competitive advantage (Kilner 1995; Cotton et al. 1999; Smiseth and Amundsen 2002). These larger and competitively superior nestlings may even beg less than their nestmates yet, for a given level of begging effort, may be more likely to receive food (Cotton et al. 1999; Parker et al. 2002; Smiseth and Amundsen 2002). Consequently, such size-related sibling competition can result in a pattern of resource allocation consistent with a nestling size hierarchy.

Parental resource allocation on the basis of offspring solicitation behavior is typical in many altricial systems; however, it is only one of three broad patterns of parentoffspring interactions (Krebs 2002). It is suggested that such parental responsiveness represents an intermediate state between the extremes of nestling control and parental control over resource distribution (Krebs 2002). In some species, most notably those that demonstrate siblicidal behavior, nestlings enforce a dominance hierarchy that regulates access to food (Mock et al. 1990; Mock and Parker 1997). Provisioning parents demonstrate little selectivity, adopting a "laissez-faire" feeding strategy and favoring dominant young (Mock 1987). Provisioning of less competitive individuals occurs as dominant individuals are fed and the ratio of need between siblings increases (Parker et al. 2002). At the other extreme, parents may demonstrate high levels of selectivity towards specific offspring, largely independent of nestling solicitation behaviors and competitive hierarchies. Such occurrences are best exemplified by patterns of solicitation and feeding in parrots (Krebs 2002).

While many avian species exhibit some degree of hatching asynchrony, the hatching period in parrots can be highly protracted due to early incubation, large clutch sizes, and longlaying intervals been eggs (Stoleson and Beissinger 1995; Krebs 2002), resulting in large size disparities between young. If parents responded to offspring solicitation behaviors or fed on the basis of dominance hierarchies, we might predict preferential feeding of larger offspring. However, in the only comprehensive studies of nestling solicitation and resource allocation in parrots, the information content of begging displays was equivocal and did not completely explain parental behavior (Stamps et al. 1989; Krebs and Magrath 2000; Krebs 2001). Furthermore, despite high hatching asynchrony and obvious size differences and competitive asymmetries between offspring, food allocation did not appear to be entirely regulated by the outcome of sibling competition. Parrot females demonstrated selective feeding of later-hatched young, while male parents favored older or larger offspring (Stamps et al. 1985; Stamps et al. 1987; Stamps et al. 1989; Krebs 2001; Krebs 2002), and such patterns of differential provisioning by parents may reflect some level of maternal compensation for hatching asynchrony.

This study contributes to our understanding of parental allocation strategies through exploration of a system hypothesized to lie at one extreme of the continuum between nestling control and parental control over resource distribution. We determine the degree of selective feeding demonstrated by male and female green-rumped parrotlets (Forpus passerinus) across asynchronous broods and explore whether provisioning strategies differ with increasing brood size. In contrast with previous parrot species studied, green-rumped parrotlets display larger clutch sizes (Budden and Beissinger 2004) and more extreme levels of hatching asynchrony, resulting in low survival of later-hatched young (Budden and Beissinger 2005). Consequently, parrotlets may be more constrained in their provisioning behaviors and prone to allocating food on the basis of offspring solicitation. Previous manipulations of hatching asynchrony have demonstrated that parents are capable of fledging as many, if not more, offspring from synchronous broods as from asynchronous broods (Stoleson and Beissinger 1997) and that supplemental feeding of laterhatched offspring greatly increases their probability of survival (Stoleson and Beissinger 1997), suggesting a strong role of sibling competition in resource acquisition. Despite increased survival of heavier eggs and a general increase in egg mass with laying sequence (Budden and Beissinger 2005), the smallest, last-hatched nestlings appear unsuccessful in acquiring parental feeds based on the results from synchrony and feeding experiments (Stoleson and Beissinger 1997). Accordingly, we might predict that, unlike female budgerigars, Melopsittacus undulatus (Stamps et al. 1985) and crimson rosellas, Platycercus elegans (Krebs 1999; Krebs et al. 1999), parrotlet parents do not selectively feed later-hatched young, making them a critical study system for interpretations of diversity in parent-offspring interactions.

Brood size was standardized as either six or eight nestlings (one above and below the mean), consistent with previous studies of parental care in this species that found large differences in nestling survival and age of fledging between these brood sizes (Stoleson and Beissinger 1997). Brood demand was also manipulated by supplementally feeding the oldest two nestlings in each brood to reduce 
their requirement for parental resources. This feeding manipulation also enabled us to evaluate the potential competitive influence of first-hatched offspring through modifying the distribution of resources among nestlings. Given our knowledge of brood reduction in this system, we predicted that (1) parental resource distribution would follow the pattern of hatching asynchrony with larger offspring receiving more feeds in both brood sizes; (2) the pattern of provisioning would not differ between parents; and (3) that any parental bias towards older offspring would be reduced following artificial supplementation of these individuals but that resource allocation across the remainder of the brood would not change.

\section{Materials and methods}

Study site and species

Data were collected between July and September 2003 from an individually color-banded population of greenrumped parrotlets nesting in Venezuela. This population has been the subject of long-term study, and further details of the site and social system are available elsewhere (Beissinger and Bucher 1992; Waltman and Beissinger 1992; Beissinger 2008).

Green-rumped parrotlets lay large clutches that hatch and fledge highly asynchronously (Stoleson and Beissinger 1997). Mean clutch size is seven eggs (range 4-12, Budden and Beissinger 2004) and females initiate incubation on the first egg (Beissinger and Waltman 1991; Grenier and Beissinger 1999). Clutches hatch over 6 to 17 days, and this high level of hatching asynchrony leads to low survival of later-hatched young (Beissinger and Waltman 1991; Stoleson and Beissinger 1997; Budden and Beissinger 2004).

Adult birds nested in 1-m deep polyvinyl chloride tubes lined with a hardware cloth (Beissinger and Bucher 1992). These nest boxes were visited daily to establish the identity of the breeding pairs and to determine clutch size, laying dates, egg sequence, and hatching and fledging dates. Eggs and nestlings were individually marked with permanent markers upon laying and hatching, respectively.

\section{Experimental setup}

We attached a small, inverted plastic cup to the bottom of the nest tube in the approximate position of the future camera on days 9-13 (first nestling hatch day=0). Parental activity was monitored to ensure continued biparental care over the next 2 days. We then replaced the whole parental nest box with a video box that was identical to the parental box with the exception of a 6-7 $\mathrm{cm}$ diameter hole cut into the tube and corresponding hardware cloth near the base.
This opening was covered by the inverted plastic cup that housed an infrared closed-circuit television (CCTV) camera pointing into the nest at $45^{\circ}$ angle. Again, parental provisioning was monitored throughout the day to ensure continued feeding.

Data collection began between days 12 and 16 (mean $14 \pm 1.2 \mathrm{SD}$ ), when the oldest nestling was halfway to fledging. On the morning of data collection, the CCTV camera was connected to a Sony GV-D800 Video Walkman recorder housed in a small water-resistant box approximately $2 \mathrm{~m}$ away. The box also contained a 12-V motorcycle battery, which powered both the camera and recording device. If parents were intolerant of either the plastic cup or video box, we abandoned the experiment, which occurred on three occasions. Following completion of the experiment, all equipment was removed, and any surrogate nestlings returned to their natal nests (see below). There were no apparent negative effects of the experimental trials on the nestlings.

\section{Experimental treatments}

\section{Brood size}

We standardized brood size as either six (ten nests) or eight nestlings (nine nests) on the basis of original clutch size in an attempt to account for variation in parental quality, so that parents provisioned an equivalent number of nestlings to their expected brood size. The mean clutch size for small broods was 6.5 eggs $( \pm 1.1 \mathrm{SD})$ and $7.6 \mathrm{eggs}( \pm 1.0 \mathrm{SD})$ for large broods; hence, cross-fostering was necessary in some instances. Given variation in hatching success, the mean natural brood size of nests assigned to the small brood treatment was 5.4 nestlings $( \pm 1.0 \mathrm{SD})$ and to large broods was 6.7 nestlings $( \pm 1.1 \mathrm{SD})$. The mean level of hatching asynchrony was 7.3 days in small broods $( \pm 1.2 \mathrm{SD})$ and 10.4 days in large broods ( $\pm 1.6 \mathrm{SD})$.

Surrogate nestlings were added the evening before data collection and remained in the nest until the end of data collection 2 days later. At this time, they were successfully returned to their natal nests, which was not part of the study. In all cases, the size of the surrogate nestling was consistent with the pattern of asynchrony in the experimental nest. Whenever possible, we tried to match the rank of the surrogate nestling within its natal nest to that of the experimental nest. Surrogate nestlings tended to be assigned to positions later in the laying sequence, and the typical donor position was "nestling 4" ( \pm 2.3 SD) in small broods and "nestling 5 " in large broods ( \pm 2.5 SD). There was no difference in the mean begging behavior measured immediately before a feeding event (see "Video analysis") of natal versus surrogate nestlings in either small or large broods (paired $t$ test; small $t_{7}=0.13, p=0.90$; large $t_{5}=0.37$, 
$p=0.73$ ) nor any difference in the number of parental feeds delivered to these nestlings (small $t_{7}=0.77, p=0.47$; large $t_{5}=0.17, p=0.87$ ).

\section{Food supplementation}

On either the first or second day of data collection, broods were assigned to a food supplementation treatment at random (four small and five large on day 1 and six small and four large on day 2). We supplemented the oldest two nestlings in the brood with a commercial diet designed for hand-rearing parrots (ROUDYBUSH ${ }^{\mathrm{TM}}$ Formula 3), using either an 18 or 20 gauge crop feeder attached to a 5 -cc syringe. Nestlings were provisioned until their crop was approximately three quarters full and still soft to the touch. Nestlings were fed immediately prior to data collection and three additional times during the 6-h data collection period (see below).

\section{Data collection}

Video data were collected beginning at first light. Nest boxes were approached while the female was still brooding and were observed until the female departed the nest between approximately 0630 and 0700 hours. Nestlings were then removed, weighed, and individually marked on the crown of the head with a nontoxic whitening solution. Nestlings 1 and 2 were fed at this time if part of the supplementation treatment. All nestlings were returned to the nest in random order, and the nest cup positioned so it was visible on camera. The recording unit was switched on and left for $90 \mathrm{~min}$, the length of the Digital 8 tapes.

Following the 90-min recording session, all nestlings were removed, and nestlings 1 and 2 were re-fed if part of the food supplementation treatment. Nestlings were again returned to the nest and a second 90-min recording session initiated. These handling periods lasted an average of $10 \mathrm{~min}( \pm 3.0 \mathrm{SD})$. This procedure was repeated twice more resulting in four 90 -min recording sessions ending between 1330 and 1400 hours. On two occasions, it was necessary to terminate data collection early due to heavy rain. At the end of the final recording session, nestlings were reweighed and returned to the nest. All video equipment was removed, and the setup procedure was repeated the following morning when the nest was subject to the alternate treatment (control or food-supplemented, as appropriate).

\section{Video analyses}

We extracted data concerning parental visits, feeding events, and nestling solicitation. For each parental visit, we recorded the start and end times to calculate visit duration. Since the camera was focused on the nest cup and not the full length of the nest cavity, these times reflected the appearance of the parent in the field of view. However, if a parent moved out of sight and returned within a minute, this was considered to be the same visit since parents rarely return to a box immediately after leaving (Waltman and Beissinger 1992).

Green-rumped parrotlets are crop feeders and provision multiple offspring during the same visit. Parents commonly occupy the center of the nest cup and select offspring by turning within the nest cup and pushing between nestlings to reach an individual (personal observation). Food transfer was identified by "beak locks," which occur when the culmen of parent and offspring are juxtaposed by approximately $90^{\circ}$ (personal observation), and "head bobs," an action present in the parent/offspring feeding unit that accompanies feeding events and is likely associated with the mechanics of food transfer (personal observation). For each visit, we recorded: (1) the number of the feeding events (within each visit for each parent); (2) the start and end times of each event to calculate duration; (3) the identity of the parent feeding and the identity of the recipient nestling; (4) the begging effort of the recipient nestling immediately before feeding (scored $0-2 ; 0$ for no begging, 1 for weak begging (nestlings gaping/head raised without accompanying body movements) and 2 for intense begging (gaping/head raised accompanied by wing flapping and up and down body movements; sensu Krebs 2001)); and (5) the begging effort of all other offspring at this time. Additional measures of offspring solicitation, such as vocalizations and response latency, were not possible due to limitations associated with this system and the fieldbased experimental design.

\section{Data analyses}

From the video, we extracted both the number and duration of feeding events as surrogates for intake volume. Within a single parental feeding visit, these variables are highly correlated with one another, in addition to each being highly correlated with change in nestling mass and perceived change in nestling crop fill (unpublished data). In this data set, the mean duration of feeds was significantly correlated with the mean number of feeds provisioned by both males and females (female $r_{19}=0.63, p=0.004$; male $r_{19}=0.64, p=0.003$ ), and there was no difference in the mean duration of a feeding event between sexes (paired $t_{15}=0.45, p=0.96$ ). Therefore, we present only data on the number of feeding events.

We used repeated measures analysis of variance to evaluate patterns of parental care, nestling behavior, and resource allocation. Analyses were conducted at two levels, either across or within broods. Brood-level analyses included brood size as a between-subjects factor in all 
cases. Parental sex and feeding manipulation were included as within-subjects (repeated) factors since parents were provisioning within the same nest and the supplemental feeding manipulation was replicated within nest. Nestling hatch order was added as a within-subjects (repeated) factor in the within-brood level analyses, and we used polynomial contrast analyses to help inform our interpretation of significant differences. Hatching time is linearly related to nestling hatch order in both small $\left(F_{1,58}=208.95, p<0.001\right.$, $\left.R^{2}=0.78\right)$ and large broods $\left(F_{1,70}=647.30, p<0.001, R^{2}=\right.$ $0.90)$. Thus, we were primarily concerned with linear contrast analyses, although we included polynomials to evaluate curvilinear effects.

When examining differences in begging behavior between artificially supplemented and nonsupplemented nestlings or between parentally fed and unfed nestlings, we used nestling "status" as a within-subjects variable. Since both of these analyses included brood size and feeding manipulation as factors in the model, we report the significance of these main effects in the first analysis only, highlighting only their interacting effects in the subsequent analysis. Nestling status is reported as a main effect in both, since it varies between the analyses.

Sample sizes for brood level analyses were ten and nine for small and large broods, respectively. Sample sizes for within-brood analyses were increased by a factor of either 6 or 8 , reflecting the number of nestlings within the broods. In both cases, samples sizes may deviate when analyzing variation between parents or across nestlings due to instances of one or the other parent not feeding.

We calculated mean begging scores per nestling from tape means and analyses of brood begging were conducted on the mean of these means. Hence, data presented are means across the $6 \mathrm{~h}$ of video data per nest. However, in six nests, there was no recording during one of the sessions due to either camera difficulties (four nests) or heavy rain (two nests). In these instances, means were calculated across the remaining tapes.

Data were analyzed using SPSS v11.0.1. Alpha was set at 0.05 throughout. Assumptions of normality, homogeneity of variance, and independence were met. When assumptions of sphericity were not supported, Huynh-Feldt adjustments were made to the degrees of freedom and $p$ values reported. Effect sizes (partial eta squared) are presented for all models, and statistical power was calculated for analyses where $0.05<p<0.10$ using $\mathrm{G}^{*}$ Power v3.0.3.

\section{Results}

Variation in parental care

The mean number of individual parental visits to the nest per hour was $1.08 \pm 0.08 \mathrm{SE}$. There was no difference in visit rate between parents $\left(F_{1,17}=0.47, p=0.50\right.$; eta $=0.03$; power $=0.10)$, brood sizes $\left(F_{1,17}=0.21, p=0.65\right.$; eta $\left.=0.01\right)$, or the feeding manipulations $\left(F_{1,17}=2.91, p=0.11\right.$; eta $=$ $0.15)$. There were no significant interactions between these factors.

Parrotlet parents fed multiple nestlings during a single visit, and the mean number of feeds per visit was $16.38 \pm$ $0.86 \mathrm{SE}$ (range 2-48). There was no difference between parents $\left(F_{1,13}=1.01, p=0.33\right.$; eta $\left.=0.07\right)$ or brood sizes $\left(F_{1,13}=2.54, p=0.13\right.$; eta $\left.=0.16\right)$ in the number of feeds provisioned and no evidence of an effect of the feeding manipulation $\left(F_{1,13}=3.47, p=0.08\right.$; eta $\left.=0.21\right)$, although power for this analysis was not high $(0.43)$, requiring an effect size of 0.25 for detection of a statistical difference. There was also no difference between brood sizes in the mean increase in nestling mass across the 2 days of the study (mass $t_{14}=0.39, p=0.70$ ).

Brood size did not affect the duration of time spent in the nest by a parent $\left(F_{1,17}=0.001, p=0.98\right.$; eta $\left.=0.01\right)$ nor were there differences between parents $\left(F_{1,17}=3.18, p=0.09\right.$; eta $=$ $0.16)$ or between supplemented and control nests $\left(F_{1,17}=\right.$ 3.54, $p=0.08$; eta=0.17). Again, these latter analyses suffered from relatively low power $(0.36$ and 0.34 respectively), requiring effect sizes of 0.19 and 0.20 for statistical differences to be detected. There were no significant interactions between any of these factors.

\section{Nestling solicitation behaviors}

There was no difference in the begging effort of nestlings in large and small broods $\left(F_{1,17}=0.49, p=\right.$ 0.49; eta=0.03; Fig. 1). Begging effort was reduced at supplemented nests $\left(F_{1,17}=13.01, p=0.002\right.$, eta $=0.43$; Fig. 1), and there was no interaction between the feeding manipulation and brood size $\left(F_{1,17}=0.01, p=0.94\right.$; eta $=$ 0.00). Supplemented nestlings (N1 and N2) exhibited reduced begging levels compared with their younger siblings $\left(F_{1,17}=25.65, p<0.001\right.$, eta $=0.60$; Fig. 1$)$, and this did not differ between brood sizes $\left(F_{1,17}=0.58, p=\right.$ 0.46; eta=0.03). As expected, there was an interaction between nestling status and the food manipulation, with supplemented nestlings reducing their begging effort following hand feeding $\left(F_{1,17}=16.90, p=0.001\right.$, eta $=$ 0.50; Fig. 1).

We examined variation in begging across nestling hatch order under control conditions in the two brood sizes separately. There was no variation in begging effort among individuals within the small brood $\left(F_{1.97,17.73}=\right.$ 0.59, $p=0.56$; eta $=0.06$; Fig. 2a). In the large brood however, older, larger nestlings begged at lower levels than their later-hatched siblings $\left(F_{4.76,38.07}=3.21, p=0.02\right.$, eta $=0.29$; linear contrast $F_{1,8}=7.20, p=0.03$; Fig. 2b). This does not appear to be a consequence of hatching 


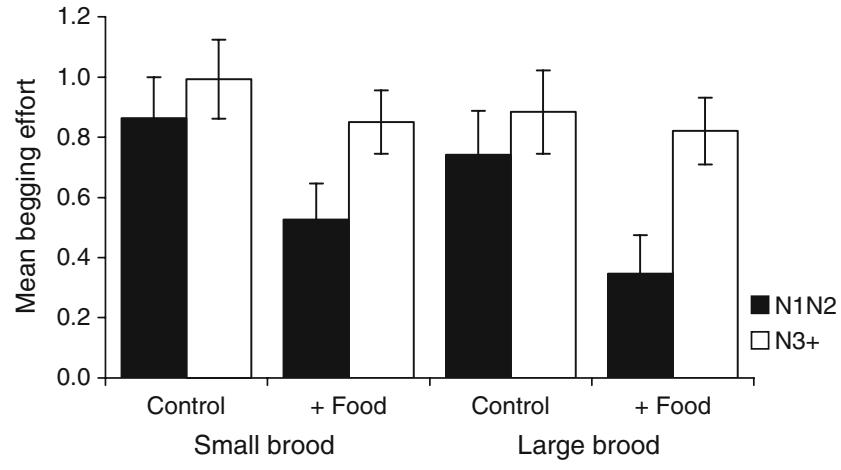

Fig. 1 Mean begging responses of first and second hatched (N1N2) nestlings versus later-hatched $(\mathrm{N} 3+)$ nestlings in small $(6 \mathrm{~N})$ and large $(8 \mathrm{~N})$ broods under control and experimental conditions. In the food supplementation treatment, nestlings $\mathrm{N} 1$ and $\mathrm{N} 2$ were hand fed prior to data collected (see "Materials and methods" for details). There were significant main effects of nestling and the food supplementation treatment and a significant interaction between the two. Data are presented as mean $\pm 1 \mathrm{SE}$

asynchrony, since there was no correlation between begging effort of older nestlings ( $\mathrm{N} 1$ and N2) and hatching asynchrony $\left(r_{19}=-0.14, p=0.56\right)$ nor between brood begging and hatching asynchrony $\left(r_{19}=-0.08, p=\right.$ $0.75)$.

\section{Parental feeding and nestling behavior}

There was no difference in brood begging according to sex of the feeding parent ("parent"; Table 1) and no significant interaction between parent and brood size (parent $\times$ brood; Table 1). Analysis of this interaction had moderate power (0.42), and an effect size of 0.24 (vs. 0.21) would have been sufficient to detect a difference. Nestlings that received food had a higher begging effort preceding the feeding event compared to siblings that were not fed ("status," Table 1; Fig. 3). This pattern did not differ between parents (status $\times$ parent; Table 1 ) or between brood sizes (status $\times$ brood; Table 1; Fig. 3). Unfed nestlings in the food addition treatment demonstrated lower begging effort than unfed nestlings in the control treatment, resulting in a significant interaction between food manipulation and nestling status (Table 1; Fig. 3). There was no interaction between parental sex and brood size with respect to the begging behavior of fed and unfed nestlings; between parental sex, food manipulation, and nestling status; nor between brood size, food manipulation, and nestling status (Table 1).

We also explored the relationship between begging effort of parentally fed nestlings and hatch order to determine the extent to which begging by nestlings of different rank may affect parental feeding. Since some offspring were not fed by one or the other parent during the 6-h observation period, we were unable to perform multi-factorial analyses and instead examined variation in begging between fed nestlings for each parent, brood size, and feeding treatment separately. Within small broods, there was no variation in begging effort according to nestling hatch order for nestlings fed by the male under control $\left(F_{3.00,15.02}=2.32\right.$, $p=0.12$; eta $=0.32 ; n=6$ ) or food manipulation treatments $\left(F_{5,25}=2.51, p=0.06\right.$; eta $=0.33 ; n=6$; power $\left.=0.49\right)$. Similarly, under neither feeding treatment was there variation in the begging effort of nestlings fed by the female (Control $F_{5,35}=0.12, p=0.99$; eta $=0.02 ; n=8$; Food Manipulation $F_{2.40,19.18}=2.05, p=0.15$; eta $\left.=0.20 ; n=10\right)$. Again, in the large brood, there was no variation in begging of offspring fed by the male under control conditions $\left(F_{7,21}=0.77, p=\right.$ 0.62 , eta $=0.20 ; n=4)$. However, it was not possible to conduct analyses on food manipulation data for male feeding events $(n=1)$. There was also no variation in begging effort of nestlings fed by the female under the control $\left(F_{7,49}=1.42, p=0.22\right.$, eta $\left.=0.17 ; n=7\right)$ or food manipulation treatments $\left(F_{7,14}=2.55, p=0.06 ; \quad\right.$ eta $=0.56$; $n=3$; power $=0.65$ ).

\section{Differential parental provisioning}

We examined the distribution of feeds across nestling hatch order within each brood size separately to determine patterns of resource allocation between parents and in response to the food manipulation.

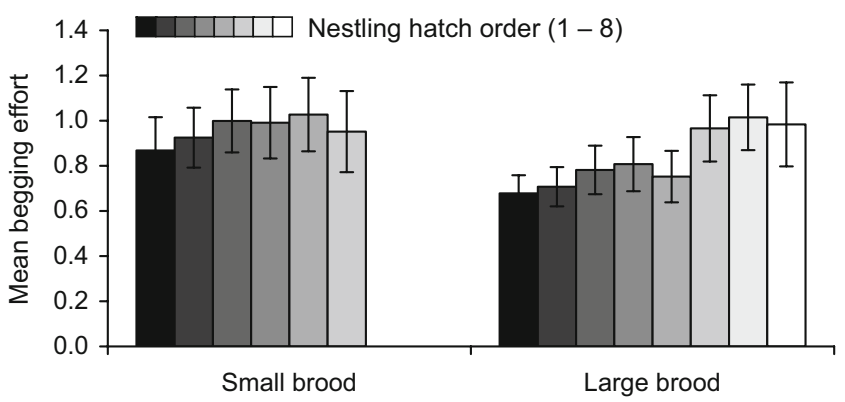

Fig. 2 Mean begging responses of individual nestlings during the control treatment in small $(6 \mathrm{~N})$ and large broods $(8 \mathrm{~N})$. Large broods show an increase in begging with hatch order, while small broods demonstrate no variation. Data are presented as mean $\pm 1 \mathrm{SE}$ 
Table 1 Repeated measures analyses of variance examining nestling begging behavior of nestlings that either did or did not receive food during a feeding event

\begin{tabular}{|c|c|c|c|c|}
\hline & $F$ & $d f$ & $p$ & Partial eta ${ }^{2}$ \\
\hline Status & 306.47 & 1,14 & $<0.001$ & 0.96 \\
\hline Parent & 0.41 & 1,14 & 0.53 & 0.03 \\
\hline Expt & 2.80 & 1,14 & 0.12 & 0.17 \\
\hline Brood & 0.84 & 1,14 & 0.37 & 0.06 \\
\hline Status $\times$ parent & 1.24 & 1,14 & 0.28 & 0.08 \\
\hline Status $\times$ expt & 15.11 & 1,14 & 0.002 & 0.52 \\
\hline Status $\times$ brood & 0.01 & 1,14 & 0.93 & 0.001 \\
\hline Parent $\times$ expt & 0.92 & 1,14 & 0.35 & 0.06 \\
\hline Parent $\times$ brood & 3.68 & 1,14 & 0.07 & 0.21 \\
\hline Expt $\times$ brood & 0.25 & 1,14 & 0.63 & 0.02 \\
\hline Status $\times$ parent $\times$ expt & 0.87 & 1,14 & 0.37 & 0.06 \\
\hline Status $\times$ expt $\times$ brood & 0.61 & 1,14 & 0.45 & 0.04 \\
\hline Status $\times$ parent $\times$ brood & 1.93 & 1,14 & 0.19 & 0.12 \\
\hline Parent $\times$ expt $\times$ brood & 0.01 & 1,14 & 0.91 & 0.001 \\
\hline Status $\times$ parent $\times$ expt $\times$ brood & 0.58 & 1,14 & 0.46 & 0.04 \\
\hline
\end{tabular}

Within subjects factors are nestling status (parentally fed/unfed; status), parental sex (parent) and experimental feeding manipulation (expt) where nestlings 1 and 2 were supplemented in experimental trials. Brood size (brood) is represented as a between subjects factor. Huynh-Feldt adjustments were made to the degrees of freedom where assumptions of sphericity were not met. Two-tailed $p$ values and effect sizes (partial eta squared) are reported

Within small broods, there was variation in the amount of food received among nestlings $\left(F_{5,45}=3.54\right.$, $p=0.01$, eta $=0.28$; Table 2). Consistent with previous results, there was no effect of supplemental feeding or parental sex on the overall number of feeds delivered and no interaction between the two (Table 2). There was an effect of the food manipulation treatment (expt $\times$ nestling; Table 2) such that nestlings 1 and 2 received less food from parents following food supplementation (Fig. 4a).

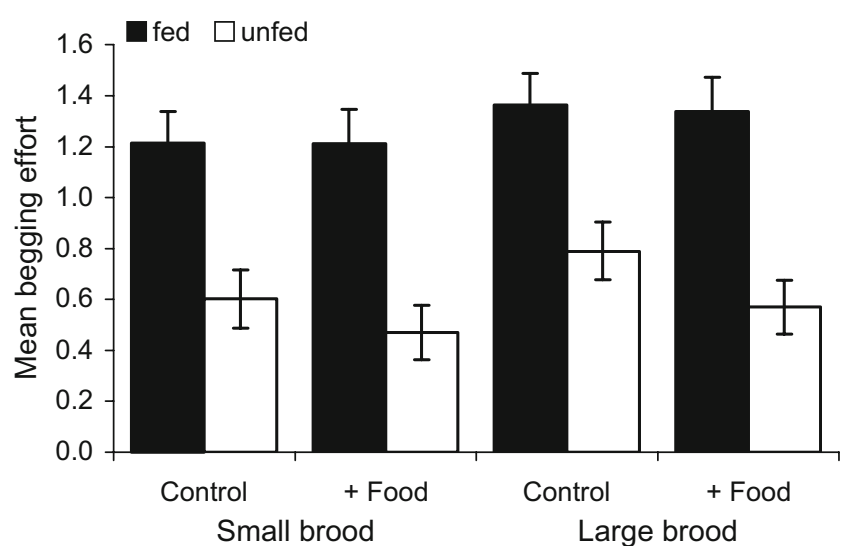

Fig. 3 Mean ( $\pm 1 \mathrm{SE})$ begging effort of nestlings immediately before parental feeding events in small and large broods. Analyses were conducted including parental sex as an interacting effect; however, only three-way interactions between nestlings status (fed/unfed), feeding treatment (control/ + food) and brood size $(6 \mathrm{~N} / 8 \mathrm{~N})$ are presented here for clarity. There were significant effects of nestling status and a significant interaction between nestling status and the feeding treatment. Data are presented as mean $\pm 1 \mathrm{SE}$
There was also an interaction between parental sex and nestling hatch order on the number of feeds received by individual nestlings (Table 2, Fig. 4a). Since parents did not differ in patterns of resource distribution in response to the food manipulation treatment (parent $\times$ expt $\times$ nestling; Table 2), we examined contrasts from analyses on control data to evaluate patterns in parental feeding. Variation in nestling provisioning was best described by linear contrast analyses $\left(F_{1,9}=4.86, p=0.05\right.$, eta $\left.=0.35\right)$; males demonstrated a reduction in feeding with nestling hatch order, while females maintained a more equitable feeding response (Fig. 4a control). This was confirmed by regression analyses of the mean number of feeds/h delivered to nestlings of different rank (male $F_{1,5}=17.38, p=$ $0.01, R^{2}=0.81$; female $F_{1,5}=0.36, p=0.58, R^{2}=0.08$ ).

Similarly in large broods, nestlings 1 and 2 received fewer parental feeds following supplementation (expt $\times$ nestling; Table 2; Fig. 4b), and overall, there was variation in the amount of feeds received among nestlings (Table 2). There was no effect of food supplementation, parental sex, or the interaction of the two on the number of feeds delivered. As in small broods, parents provisioning large broods varied in the allocation of resources among nestlings (parent $\times$ nestling; Table 2). Again, we examined contrasts from analyses on control data to evaluate patterns in parental feeding across the brood. Variation in nestling provisioning was again best described by linear contrast analyses $\left(F_{1,8}=14.91, p=0.005\right.$, eta $\left.=0.65\right)$, with males demonstrating a general decrease in feeding with nestling hatch order, while females increased feeding with 
Table 2 Repeated measures analyses of variance examining distribution of resources (feeds/h) across nestlings in small and large broods separately

\begin{tabular}{|c|c|c|c|c|}
\hline & $F$ & $d f$ & $p$ & Partial eta ${ }^{2}$ \\
\hline \multicolumn{5}{|l|}{ Small brood (6 nestlings) } \\
\hline Nestling & 3.54 & 5,45 & 0.01 & 0.28 \\
\hline Expt & 0.02 & 1,9 & 0.90 & 0.002 \\
\hline Parent & 0.18 & 1,9 & 0.68 & 0.02 \\
\hline Parent $\times$ expt & 0.06 & 1,9 & 0.82 & 0.01 \\
\hline Expt $\times$ nestling & 10.06 & 5,45 & $<0.001$ & 0.52 \\
\hline Parent $\times$ nestling & 6.43 & $2.98,26.87$ & 0.002 & 0.42 \\
\hline Parent $\times$ expt $\times$ nestling & 1.00 & 5,45 & 0.43 & 0.10 \\
\hline \multicolumn{5}{|l|}{ Large brood (8 nestlings) } \\
\hline Nestling & 3.01 & 7,56 & 0.01 & 0.27 \\
\hline Expt & 0.17 & 1,8 & 0.69 & 0.02 \\
\hline Parent & 1.93 & 1,8 & 0.20 & 0.19 \\
\hline Parent $\times$ expt & 1.53 & 1,8 & 0.25 & 0.16 \\
\hline Expt $\times$ nestling & 4.11 & 7,56 & 0.001 & 0.34 \\
\hline Parent $\times$ nestling & 4.19 & 7,56 & 0.001 & 0.34 \\
\hline Parent $\times$ expt $\times$ nestling & 0.70 & 7,56 & 0.68 & 0.08 \\
\hline
\end{tabular}

Within-subjects factors explaining resource distribution are nestling hatch order (1-6 or 1-8; nestling), parental sex (parent) and experimental feeding manipulation (expt) where nestlings 1 and 2 were supplemented in experimental trials. Huynh-Feldt adjustments were made to the degrees of freedom where assumptions of sphericity were not met. Two-tailed $p$ values and effect sizes (partial eta squared) are reported

hatch order except for the last-hatched offspring (Fig. 4b). Again, regression analyses of the mean number of feeds delivered to nestlings of different rank by males confirmed this pattern $\left(F_{1,7}=12.74, p=0.01, R^{2}=0.68\right)$. However, due to the reduced feeding of last-hatched young by females, regression across all nestlings was not significant $\left(F_{1,7}=\right.$ 1.91, $\left.p=0.22, R^{2}=0.24\right)$. Removal of this last individual revealed a strong increase in maternal feeding with nestling hatch order across nestlings $1-7 \quad\left(F_{1,6}=20.50\right.$, $\left.p=0.01, R^{2}=0.80\right)$.

a Small broods $(6 \mathrm{~N})$

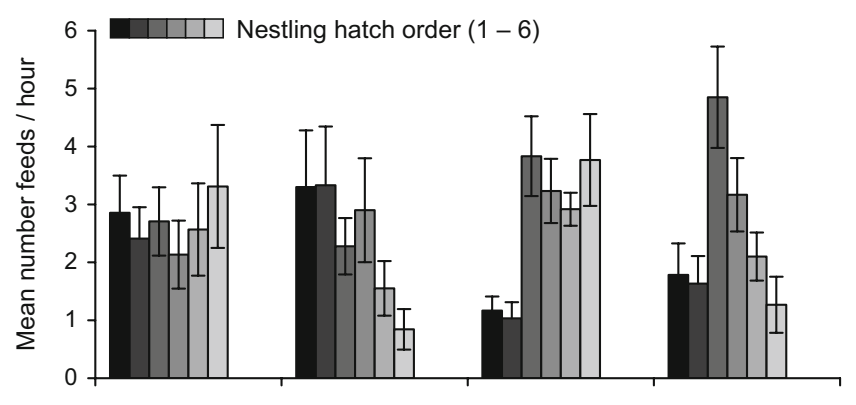

b Large broods $(8 \mathrm{~N})$

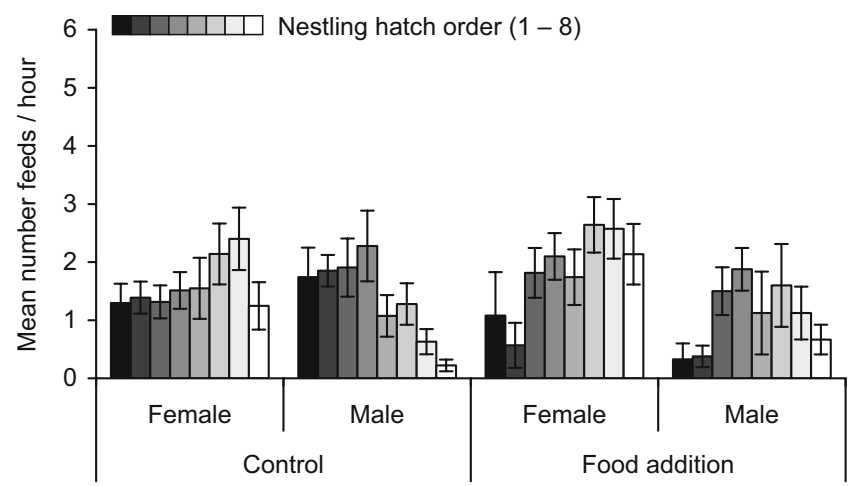

Fig. 4 Mean $( \pm 1$ SE) number of feeds received by nestlings per hour from male and female parents. Data are presented separately for a small $(6 \mathrm{~N})$ and $\mathbf{b}$ large broods $(8 \mathrm{~N})$ with variation between parents and according to the experimental feeding manipulation shown 


\section{Discussion}

Variation in brood level provisioning and resource limitation

There was no apparent difference between male and female green-rumped parrotlets in brood level provisioning; parents had similar visit rates per hour and engaged in a comparable number of feeding events per visit. Visit rates were consistent across small and large broods, indicating that parents do not respond to increased brood demand through an increase in the number of provisioning events, although we observed a trend for reduced feeding events per visit when provisioning food supplemented broods. That there was no difference in provisioning between the two brood sizes may suggest that larger broods were more food limited than smaller broods. However, there was also no discernible difference in the mean mass gain of nestlings between the two brood sizes, and begging levels were also similar. Consequently, this finding may reflect variation in the quality of parents assigned to the two treatment groups, since brood size was standardized on the basis of original clutch size. Higher quality parents laying larger clutches may have the potential to adjust load sizes delivered to nestlings, and additional data would be required to confirm this. Nevertheless, patterns of brood reduction in this system, where the probability of survival decreases with increasing brood size, are consistent with the observed equitable delivery rates demonstrated in this study (Beissinger and Waltman 1991; Stoleson and Beissinger 1997; Budden and Beissinger 2004).

\section{Begging in parrotlets}

The food manipulation treatment resulted in a decrease in brood begging, driven largely by the reduction in begging effort of the supplemented nestlings, and suggested that begging in parrotlets varies with hunger in a meaningful way. Furthermore, begging effort was higher in nestlings that received food than those that did not, suggesting parrotlet parents provision on the basis of begging behavior. Yet adults did not reduce their visit rate in response to less vigorous brood begging. Krebs (2002) suggested begging rates in parrots can be highly variable among broods and do not necessarily relate to hunger in a simple way. For example, brood begging levels in budgerigars are positively correlated with feeding rates (Stamps et al. 1989), while in crimson rosellas, hungry nestlings do not beg more than their siblings, and adults do not increase their feeding rates with increased begging (Krebs and Magrath 2000; Krebs 2001). It is possible that variation in begging more accurately reflects variation in parental responses to nestlings rather than nestling responses to hunger (Krebs 2002), and it is interesting that large broods tended to beg more in the presence of a female parent (an additional four nests would have been necessary for detection of a statistical difference). Moreover, parrotlet parents may use multiple cues to regulate their provisioning rates, including nestling crop size which was not accurately discernible on video, and the single measure of begging used in this study may not provide the degree of variation necessary to infer subtle changes in offspring hunger.

We found that smaller, last-hatched green-rumped parrotlet nestlings begged at greater levels than their older siblings, as in other parrot systems (Stamps et al. 1989; Krebs 2001). However, this was only true in large broods and largely resulted from a reduction in begging by older nestlings rather than an increase in begging by younger individuals. This pattern has also been observed in a number of non-psittacine species exhibiting asynchronous hatching (Price and Ydenberg 1995; Lotem 1998; Cotton et al. 1999), and the prediction that large (competitively superior) individuals are able to maintain their share of resources with lower investment in begging (Parker et al. 1989) is supported empirically (Cotton et al. 1999). However, differences in begging between the two brood sizes may also reflect ontogenetic differences. Given the degree of hatching asynchrony and resulting variation in nestling ages between brood sizes, in this study, large parrotlet broods were, on average, younger than small broods. Krebs (2001) found that variation in nestling begging was more pronounced in younger rosella broods, so the observed pattern may be attributable to nestling development rather than competition arising from increased brood size.

Distribution of resources across nestling hatch order

There was significant variation in provisioning among nestlings in both brood sizes, and not surprisingly, the two supplemented nestlings were fed less by parents during the food manipulation treatment. While parents did not appear to respond differently to brood level hunger, they did demonstrate differences in the provisioning of individual offspring.

Male parrotlets biased their feedings towards older or earlier-hatched offspring, which is consistent with feeding patterns in both crimson rosellas and budgerigars (Stamps et al. 1985; Krebs 1999). We found no evidence of increased begging by older nestlings, suggesting that males actively favor larger offspring or provision according to the outcome of sibling competition. The competitive advantage afforded by increased size may enable larger nestlings to occupy positions closer to the provisioning parent or reach higher towards the adult. Proximity is important in securing parental resources in a number of species (Malacarne et al. 1994; 
Kacelnik et al. 1995; Cotton et al. 1999; Whittingham et al. 2003), and nestlings have been shown to jostle one another in order to obtain a location favored by the provisioning parent (Gottlander 1987; McRae et al. 1993; Kolliker et al. 1998; Kolliker and Richner 2004). However, if size-related sibling competition is important in driving resource allocation, why is the pattern of female provisioning not consistent with nestling size hierarchy as in males?

The feeding patterns demonstrated by adult female parrotlets was similar to variation in offspring begging in both brood sizes, with increased feeding of later-hatched offspring in large broods. However, the last-hatched offspring in these broods received less food than would be predicted by their begging behavior, and the converse was true for small broods, suggesting that females are not responding to solicitation alone. Such active food allocation towards individual offspring may reflect female compensation for the constraints imposed by hatching asynchrony. Increased food allocation to last-hatched young increases the probability of survival in this species (Stoleson and Beissinger 1997), and selective feeding of smaller offspring decreases the potential for large individuals to monopolize resources, reducing sibling competition and increasing growth and survival (Krebs et al. 1999). Interestingly, mean levels of provisioning in large broods varied in a manner similar to patterns of female allocation, suggesting that females were more than able to compensate for the reduced provisioning of younger offspring by males. Even so, feeds directed to the youngest offspring were still reduced in comparison with feeds to its closest older sibling.

Selective feeding of small offspring by mothers has been observed in other birds (Gottlander 1987; Leonard and Horn 1996; reviewed in Slagsvold 1997), including parrots (Stamps et al. 1985; Krebs et al. 1999; Krebs 2002). It has been suggested that parental differences in the costs incurred during care-giving may result in provisioning biases (Lessells 2002; Hamer et al. 2006). If provisioning small young requires additional time by parents, then this may reflect a significant cost to those parents that attend the nest less frequently. Conversely, increased time at the nest (due to brooding or other behaviors) may allow females to more accurately discriminate variation in the needs of offspring (Gottlander 1987; Krebs 1999). Although we did not detect a statistical difference in the time spent at the nest by females versus males $\left(F_{1,17}=3.18, p=0.09\right.$; eta $\left.=0.16\right)$ or in the time parents spent at food-supplemented nests $\left(F_{1,17}=3.54, p=\right.$ 0.08 ; eta $=0.17$ ), the power of these analyses was relatively low. An additional seven or nine nests would have been required for detection of statistical differences between parents or by the food manipulation treatment, respectively. However, these results may suggest that a bias away from provisioning larger offspring or a female bias towards younger offspring requires additional time.
If increased feeding of younger offspring reduces the occurrence of brood reduction, then why do female parents of small broods not also provision in a manner that enhances brood survival? First, the feeding patterns observed in small broods may not necessarily result in significant brood reduction. Younger, smaller nestlings are unlikely to require as many resources as their older nestmates given their reduced size, and the fledging success of the last nestlings in a six-nestling brood is not significantly lower than that of a number of their older nestmates despite variation in fledging success (Budden and Beissinger 2004). Thus, the apparent female compensation for nestling size asymmetry that occurs primarily in large broods suggests such differential resource allocation may only be necessary when the probability of survival for later-hatched young is significantly reduced. Whether the observed differences in provisioning patterns between parents of small and large broods reflects facultative adjustment or variation in parental quality requires further consideration. Nonetheless, our current understanding of parental and offspring control over resource allocation would benefit from additional research on alternate model systems, such as parrots.

Acknowledgments We are grateful to Catherine Dana and Rebecca Miller for assistance in conducting the field experiments and to Catherine Dana and Timothy Bonebrake for analysis of video tapes. We also wish to thank Tomas Blohm, Malu Gonzales, and Jessica Blickley for important contributions to the data collection and logistics in Venezuela, and Letitia Grenier for valuable discussions of this work. This work was supported by a grant to SRB from the National Science Foundation and a Carl B. Koford Memorial Fund Research Grant to AEB. This research was conducted in accordance with Venezuelan regulations.

Open Access This article is distributed under the terms of the Creative Commons Attribution Noncommercial License which permits any noncommercial use, distribution, and reproduction in any medium, provided the original author(s) and source are credited.

\section{References}

Beissinger SR (2008) Long-term studies of the Green-rumped Parrotlet (Forpus passerinus) in Venezuela: hatching asynchrony, social system and population structure. Ornitol Neotrop 19:73-83

Beissinger SR, Waltman JR (1991) Extraordinary clutch size and hatching asynchrony of a neotropical parrot. The Auk 108:863871

Beissinger SR, Bucher EH (1992) Can parrots be conserved through sustainable harvesting. Bioscience 42:164-173

Beissinger SR, Tygielski S, Elderd B (1998) Social constraints on the onset of incubation in a neotropical parrot: a nestbox addition experiment. Anim Behav 55:21-32

Beissinger SR, Cook MI, Arendt WJ (2005) The shelf life of bird eggs: testing egg viability using a tropical climate gradient. Ecology 86:2164-2175

Bollinger PB, Bollinger EK, Malecki RA (1990) Tests of 3 hypotheses of hatching asynchrony in the common tern. Auk 107:696-706 
Budden AE, Wright J (2001) Begging in nestling birds. Curr Ornithol 16:83-118

Budden AE, Beissinger SR (2004) Against the odds? Nestling sex ratio variation in green-rumped parrotlets. Behav Ecol 15:607613

Budden AE, Beissinger SR (2005) Egg mass in an asynchronously hatching parrot: does variation offset constraints imposed by laying order? Oecologia 144:318-326

Clark AB, Wilson DS (1981) Avian breeding adaptations: hatching asynchrony, brood reduction and nest failure. Q Rev Biol 56:253-277

Cook MI, Beissinger SR, Toranzos GA, Rodriguez RA, Arendt WJ (2005) Microbial infection affects egg viability and incubation behavior in a tropical passerine. Behav Ecol 16:30-36

Cotton PA, Wright J, Kacelnik A (1999) Chick begging strategies in relation to brood hierarchies and hatching asynchrony. Am Nat 153:412-420

Godfray HCJ (1991) Signalling of need by offspring to their parents. Nature 352:328-330

Godfray HCJ (1995) Signaling of need between parents and young: parent-offspring conflict and sibling rivalry. Am Nat 146:1-24

Gottlander K (1987) Parental feeding behaviour and sibling competition in the pied flycatcher Fidecula hypoleuca. Ornis Scandinavica 18:269-276

Grenier JL, Beissinger SR (1999) Variation in the onset of incubation in a neotropical parrot. Condor 101:752-761

Hamer KC, Quillfeldt P, Masello JF, Fletcher KL (2006) Sex differences in provisioning rules: responses of Manx shearwaters to supplementary chick feeding. Behav Ecol 17:132-137

Kacelnik A, Cotton PA, Stirling L, Wright J (1995) Food allocation among nestling starlings: sibling competition and the scope of parental choice. Proc R Soc Lond B Biol Sci 259:259-263

Kilner R (1995) When do canary parents respond to nestling signals of need? Proc R Soc Lond B Biol Sci 260:343-348

Kolliker M, Richner H (2004) Navigation in a cup: chick positioning in great tit, Parus major, nests. Anim Behav 68:941-948

Kolliker M, Richner H, Werner I, Heeb P (1998) Begging signals and biparental care: nestling choice between parental feeding locations. Anim Behav 55:215-222

Krebs EA (1999) Last but not least: nestling growth and survival in asynchronously hatching crimson rosellas. J Anim Ecol 68:266-281

Krebs EA (2001) Begging and food distribution in crimson rosella (Platycercus elegans) broods: why don't hungry chicks beg more? Behav Ecol Sociobiol 50:20-30

Krebs EA (2002) Sibling competition and parental control: Patterns of begging in parrots. In: Wright J, Leonard ML (eds) The evolution of begging: competition, cooperation and communication. Kluwer Academic, Dordrecht, pp 319-336

Krebs EA, Magrath RD (2000) Food allocation in crimson rosella broods: parents differ in their responses to chick hunger. Anim Behav 59:739-751

Krebs EA, Cunningham RB, Donnelly CF (1999) Complex patterns of food allocation in asynchronously hatching broods of crimson rosellas. Anim Behav 57:753-763

Leonard M, Horn A (1996) Provisioning rules in tree swallows. Behav Ecol Sociobiol 38:341-347

Lessells CM (2002) Parentally biased favouritism: why should parents specialize in caring for different offspring? Philos Trans R Soc Lond B Biol Sci 357:381-403
Lotem A (1998) Higher levels of begging behavior by small nestlings: a case of a negatively correlated handicap. Isr J Zool 44:29-45

Magrath RD (1990) Hatching asynchrony in altricial birds. Biol Rev 65:587-622

Malacarne G, Cucco M, Bertolo E (1994) Sibling competition in asynchronously hatched broods of the Pallid Swift (Apus pallidus). Ethol Ecol Evol 6:293-300

McRae SB, Weatherhead PJ, Montgomerie R (1993) American robin nestlings compete by jockeying for position. Behav Ecol Sociobiol 33:101-106

Mock D, Parker G (1997) The evolution of sibling rivalry. Oxford University Press, Oxford

Mock DW (1987) Siblicide, parent-offspring conflict, and unequal parental investment by egrets and herons. Behav Ecol Sociobiol 20:247-256

Mock DW, Drummond H, Stinson CH (1990) Avian siblicide. Am Sci 78:438-449

Muller W, Lessells M, Korsten P, von Engelhardt N (2007) Manipulative signals in family conflict? On the function of maternal yolk hormones in birds. Am Nat 169:E84-E96

Nilsson JA (1995) Parent-offspring interaction over brood sizecooperation or conflict. J Avian Biol 26:255-259

Parker GA, Mock DW, Lamey TC (1989) How selfish should stronger sibs be? Am Nat 133:846-868

Parker GA, Royle NJ, Hartley IR (2002) Begging scrambles with unequal chicks: interactions between need and competitive ability. Ecol Lett 5:206-215

Price K, Ydenberg R (1995) Begging and provisioning in broods of asynchronously-hatched yellow-headed blackbird nestlings. Behav Ecol Sociobiol 37:201-208

Romagnano L, Hoffenberg AS, Power HW (1990) Intraspecific brood parasitism in the European starling. Wilson Bulletin 102:279-291

Slagsvold T (1997) Brood division in birds in relation to offspring size: sibling rivalry and parental control. Anim Behav 54:1357-1368

Smiseth PT, Amundsen T (2002) Senior and junior nestlings in asynchronous bluethroat broods differ in their effectiveness of begging. Evol Ecol Res 4:1177-1189

Stamps JA, Clark A, Arrowood P, Kus B (1985) Parent-offspring conflict in budgerigars. Behaviour 94:1-40

Stamps JA, Clark A, Kus B, Arrowood P (1987) The effects of parentoffspring gender on food allocation in budgerigars. Behaviour 101:177-199

Stamps JA, Clark A, Arrowood P, Kus B (1989) Begging behavior in budgerigars. Ethology 81:177-192

Stoleson SH, Beissinger SR (1995) Hatching asynchrony and the onset of incubation in birds revisited. When is the critical period? Curr Ornithol 12:191-270

Stoleson SH, Beissinger SR (1997) Hatching asynchrony, brood reduction, and food limitation in a neotropical parrot. Ecol Monogr 67:131-154

Stoleson SH, Beissinger SR (1999) Egg viability as a constraint on hatching synchrony at high ambient temperatures. J Anim Ecol 68:951-962

Veiga JP (1992) Hatching asynchrony in the house sparrow-a test of the egg-viability hypothesis. Am Nat 139:669-675

Waltman JR, Beissinger SR (1992) Breeding-behavior of the greenrumped parrotlet. Wilson Bull 104:65-84

Whittingham LA, Dunn PO, Clotfelter ED (2003) Parental allocation of food to nestling tree swallows: the influence of nestling behaviour, sex and paternity. Anim Behav 65:1203-1210 